Research Article

\title{
A Dynamic Day-To-Day Departure Time and Route Choice Model for Bounded-Rational Individuals
}

\author{
Lingjuan Chen $\mathbb{D}^{1},{ }^{1}$ Yu Wang $\mathbb{D}^{1},{ }^{1}$ and Dongfang $M a \mathbb{D}^{2}$ \\ ${ }^{1}$ School of Automobile and Traffic Engineering, Wuhan University of Science and Technology, Wuhan, China \\ ${ }^{2}$ Institute of Marine Information Science and Technology, Zhejiang University, Hangzhou 310058, China \\ Correspondence should be addressed to Dongfang Ma; mdf2004@zju.edu.cn
}

Received 9 December 2020; Revised 9 March 2021; Accepted 24 March 2021; Published 8 April 2021

Academic Editor: Zeshui Xu

Copyright ( 92021 Lingjuan Chen et al. This is an open access article distributed under the Creative Commons Attribution License, which permits unrestricted use, distribution, and reproduction in any medium, provided the original work is properly cited.

Accurate prediction of travellers' day-to-day departure time and route choice is critical in advanced traffic management systems. There have been several related works about route choice with the assumption that the departure time for individual travellers is known beforehand. With real-time traffic state information provided by navigation systems and previous historical experience, travellers will dynamically update their departure time, which is neglected in existing works. In this study, we aim to describe travellers' spatial-temporary choice behaviour taking navigation information into account and propose a bounded-rational day-to-day dynamic learning and adjustment model. The new model contains three steps. First, the real-time navigation guidance on each discrete day is obtained, and the self-learned experience of travellers' choices with navigation information is presented; then, the day-to-day revision process of the choices is derived to maximize departure and route choice prospect; next, by aggregating each individual's behaviour and calculating route choice probability, a bounded-rational continuous day-to-day dynamic model is provided. Numerical experiments suggest that the proposed model converges to a spatial-temporal oscillating equilibrium not a fixed-point stable status, and the final equilibrium trend is different from classical user equilibrium. The findings of the study are helpful to improve the prediction accuracy of traffic state in urban street networks.

\section{Introduction}

Forecasting the spatial-temporal distribution of traffic flow during a certain period is crucial in intelligent transportation systems (ITS). For example, authorities can optimize traffic organization schemes and signal timing plans based on forecasting results. There has been a large number of related works about traffic flow forecasting, including short-term and daily traffic flow forecasting by mining changing regular patterns from historical data. These methods just extract the representation of these patterns but do not explore the root causes of changes.

For a given traffic demand, the spatial-temporal distribution of vehicles across the whole network is the result of traffic flow assignment. However, traditional flow assignment models are static or dynamic network flow equilibria, which focus on final states while overlooking long oscillation processes present in the flow. Thus, a precise description of the traffic flow assignment dynamic process is crucial to determine the spatial-temporal distribution of vehicles.

User equilibria (UE) or stochastic user equilibria (SUE) are long-term dominant assignment models that formulate a final static state under the hypothesis that each traveller is perfectly rational and has access to real-time information about the whole network $[1,2]$. However, it is obvious that this hypothesis is too idealistic to correspond to reality and some deficiencies exist. For example, navigation apps, traffic radio, self-experience, or friends' shared data are familiar sources regarding travel time, queue length, or congestion, but access to each link's complete real-time information is not always available. Hence, UE or SUE's preconditions are generally not guaranteed. In addition, the overwhelming majority shows reliance on past experience and refuses to make changes unless an obvious better choice appears, and the difference is beyond users' tolerance. In other words, users show bounded rationality $[3,4]$. These issues are 
evident in daily traffic flow assignment models; cyclical or concussive day-to-day equilibria are possible over relatively long periods. Furthermore, precise forecasting is extremely difficult due to individuals' day-to-day dynamic adjustments to comprehensive influence factors. For example, users revise their departure time and route day by day based on selfexperience and real-time information. This indicates that describing the spatial-temporal daily flow variation and finding a stable equilibrium is very important. However, the equilibrium models only focus on the final conditions, ignoring the day-to-day adjusting process. Therefore, modelling day-to-day dynamic behaviour incorporating choice psychology and information from various sources is the foundation for forecasting spatial-temporal flow distribution accurately.

Research on day-to-day dynamic assignment models has a long history, which dates back to the contributions of [5-9]. These works suggest that day-to-day dynamics are a question of how users adjust their route choice to reach a stable state and propose mathematical models to describe the process. Then, this research area has seen some further development, and some key themes include (1) establishing equilibrium models similar to daily models: adjustment process, dynamic user equilibria, or bounded rational user equilibria; (2) equilibrium and stability analysis: if the dayto-day model converges or not, how long the oscillations last and what is the final stable state? There are different works presented in literature which focus on day-to-day traffic assignment models and solutions' theoretical properties. A comprehensive review and presentation of these works is presented in [10].

A precise description of travellers' day-to-day adjustment behaviour under realistic network environments is foremost in constructing day-to-day dynamic models. However, at the present stage, it is not known which factors are involved in the adjustment process and how to combine factors to form a unified framework. Furthermore, criteria are expected to differ among individuals. Hence, attention should be paid to the state assumptions on users' behaviour and clarify these influence factors. Three important points of concerns can be considered:

(1) Information sources, data collection methods, and users' information processing modes: users seek other path options whose utility is better than the experienced one to revise their choice. Information about route travel time, flow density, and so on of a certain alternative should be accessible. There are many devices and sources used for collecting realtime or forecasted travel information. The most wellknown source is ATIS, which provides travel cost and has a great impact on day-to-day flow evolution. An extensive attention has been paid to establish the mathematical model to describe the users' learning method and flow evolution process with the assumption that users perceive each route's historical travel time [11-14]. Furthermore, the forecasted information is another focus with the assumption that ATIS provides estimated travel time in the coming day. The literature in this field includes the discussion of how the information accuracy and ATIS penetration influence the travellers' learning process and models' stability [15-17]. In our work, instead of investigating the forecast accuracy, we concentrate on day-to-day and within-day flow evolution models to explicitly explain the characteristics with bounded rationality. Therefore, the absolute historical information is assumed as users' perceived travel time and the learning process.

However, it is notable that the historical information significantly influences the predeparture choice. With the popularity of smart phones and $4 \mathrm{G} / 5 \mathrm{G}$ networks, navigation applications can provide routes' real-time enroute travel costs and a portion of travellers abandon the pretrip choice. In these respects, several studies have shown that traffic sensors and mobile navigation systems will fundamentally change the mode and type of traffic information collection, allowing GPS-enabled mobile devices to be utilized in estimating traffic states [18-20]. Contrary to the historical data, the real-time enroute information has no impact on the departure time within-day, users' self-experience learning, and perceived information. In general, users choose a preferred pretrip route and departure time with the utilization of historical information and switch to the shorter routes recommended by the navigation system. For the above discussion, navigation application and historical experience is the focus of the present study, which aims to describe information collection behaviour and day-to-day adjustment models.

(2) Users' risk attitude on uncertainties and alternatives: in UE- or SUE-based methods, each traveller is assumed to be perfectly rational. In other words, users always choose the route which has the greatest utility. In reality, however, for day-to-day behaviour revision, the majority of users show bounded rationality and relies on paths which are frequently adopted unless the time cost or congestion is beyond individuals' tolerance, and a distinctly improved alternative can obtained. Many related studies have also suggested that travellers could take any route whose total travel cost is within an "indifference band" of the optimal path cost $[21,22]$. Therefore, users show bounded rational behaviour when responding to reality.

(3) How do travellers coordinate departure time and route choice? Do the two processes need to satisfy the same criteria? Do the users make these choices simultaneously? Many researchers have dedicated themselves to these topics, and a majority of noteworthy studies and important conclusions can be found in [23-26]. However, in various models, time choice is just regarded as an element of the routes' utility, integrated in the route choice model but not forming an independent adjustment model. In this 
study, departure time is described as an independent course to maximize different utility.

From the above three points, the purpose of the present study is to propose a bounded-rational day-to-day dynamic model which explicitly combines departure time adjustment and route choice based on the historical and real-time information. The formulated model will illustrate the process of individual information collection, experience utilization, choice revision, and the spatial-temporal distribution of vehicles on each day. It also enables us to comprehend how the travellers' tolerance, bounded rationality, and information reliance affect the convergence and final stability.

The rest of this study is structured as follows. In Section 2 , we explain how individuals collect information and individuals' choice propensity in day-to-day behaviour. In Section 3, we formulate a microscopic model to illustrate individuals' information processing modes and day-to-day revision regulation. In Section 4, the microscopic individual model is transformed into an aggregated one, which enables us to observe how the flow is distributed spatially and temporally. In Section 5, the model's theoretical properties are discussed. In Section 6, we propose a numerical example for comparative experiments to validate the model, explicitly presenting the flow distribution feature, and examine how navigation information affects convergence. In Section 7, we summarize the study and present some directions for future research.

\section{Microscopic Analysis of Day-To-Day Behaviour Adjustment}

In this section, we present an elaborate description for users' day-to-day behaviour, which incorporates properties of individuals' information collection, the method of alternative routes' travel time determination, and principles of daily departure time and route adjustment over the system's evolution. The model proposed in this study aims to represent a dynamic system which consists of commuters who choose an alternative from an option set day-by-day. The formulation of the model and the details of the system are determined by the following assumptions:

(1) The system evolves as the days pass. The proposed day-to-day model is coupled with a within-day model which calculates travel time for each link and describes users' adjustment behaviour when they acquire information. An integer $d$ is adopted to describe the date of the system. For dynamic features of the within-day model, the peak hour for commuters is divided into identical segments denoted by $k(k=1,2, \ldots, K)$. Therefore, the dynamics of realtime flow are approximated using a quasidynamic model by minimizing each segment's duration.

(2) Utility theory is traditionally applied in travel behaviour modelling. However, for the consideration of the network's uncertainties and users' risk propensity, stochastic utility value does not encompass individuals' properties and has nonnegligible shortcomings for describing system randomness. Cumulative prospect theory $[27,28]$ is based on the travel time's probability density function and distribution function. Therefore, it has the advantage of describing the users' risk propensity when they encounter the system's randomness.

(3) Travellers collect information from navigation applications and past travel memory. To conform to reality, the first two options that have shorter travel times are recommended to users who compare each alternative's cost and select the one with the greatest prospective value.

(4) Principles of departure time and route choice: for users, the departure time and budget planning based on the need for on-time arrival to their destination. In fact, travel time is a random variable obeying some kinds of probability distribution and on-time arrival is, strictly speaking, impossible. For commuters, arriving late is not allowed, while arriving too early is not cost-effective; therefore, arriving within a fixed time window around the work start time is satisfactory and tantamount to on-time arrival. Because of the system's statistics, the only feasible goal is to increase the on-time probability in the time window. Accompanying the first step that determines the departure time, route choice based on navigation guidance is followed closely to minimize actual travel time.

(5) The explicit process of dynamic day-to-day adjustment is assumed to comply with the following hypothesis; first of all, individuals can measure their travel time at the end of each day and update the perceived travel time day by day via limited memory; second, users choose a path with a preselected budget time; in other words, users determine their departure intervals to avoid later penalties, then choose the route to pursue the largest prospect value. Finally, with real-time navigation information during their departure interval, a portion of users will convert to the recommended paths from their preselected ones.

We focus on the design of the mathematical model and the theoretical properties. The contribution and improvements of this work are summarized.

(1) When formulating the dynamic model, the "dynamic" includes two aspects, i.e., day-to-day evolution and the discrete departure time fluctuation within day. In this work, various decision targets and the interrelationship of the two dynamics, which are overlooked in previous literature, are discussed separately.

(2) The revision flow is proportional to the original flow and bounded limit, instead of UE or SUE equilibrium assignment. This evolution process generates some distinctive model and solution characters. 


\section{Individuals' Microscopic Choice Model}

3.1. Self-Learning Model. The road network is denoted as $G(N, A)$, where $N$ is the set of nodes and $A$ is the set of links. $W$ represents the set of origin-destination (OD) pairs; $R_{w}$ is the routes' set for OD pair $w \in W$, and we denote $N_{w}$ as the total number of paths. Free travel time, travel time, link flow, and the capacity of link $a \varepsilon A$ is described by the variables $t_{a}^{0}$, $t_{a}, x_{a}$, and $c_{a}$, respectively. The route $r$ denotes a set of links $a 1, a 2, \ldots$, route travel time $t_{r}=\sum_{\forall a} \delta_{a, r} \times t_{a}$, if $a \varepsilon r, \delta_{a, r}=1$; otherwise, $\delta_{a, r}=0$.

The perceived travel time $T_{k, r}^{d}$ of route $r$ selected by users at the current day $d$ and interval $k$ is stored after finishing the journey. In correspondence with the Ebbinghaus forgetting curve, the memory's influence of experienced travel time is decreasing as the system evolves [29, 30]. Meanwhile, a threshold of $L$ days is also set for memory storage. In other words, only experience within $l$ days, where $d-L \leq l<d$, is taken into account for the perceived travel time at the current day $d$. A decreasing linear function between memory impact degree $y_{d-l}$ and the number of elapsed days $l$ is defined as $y_{d-l}=F(l)$. Therefore, the user's perceived travel time $T_{k, r}^{d+1}$ for route $r$ can be expressed as follows:

$$
T_{k, r}^{d+1}=\frac{\left(\sum_{l=0}^{L-1} T_{k, r}^{d-l} \times y_{d-l}\right)}{\sum_{l=0}^{L-1} y_{d-l}}, \quad \forall r \in R_{w} .
$$

Therefore, if $d-L>l$, the past travel memory on day $l$ is considered to have faded and has no impact on $T_{k, r}^{d+1}$.

Some researchers have stated that the route travel time can be regarded as a stochastic variable and can be approximated using a normal distribution according to law of large numbers. Therefore, denoting the route travel time at day $d$ and interval $k$ as variable $t_{k, r}^{d}$, this assumption can be written as

$$
t_{k, r}^{d} \sim N\left(\tau_{k, r}^{d}, \sigma_{k, r}^{d}\right)
$$

In equation (2), $\tau_{k, r}^{d}, \sigma_{k, r}^{d}$ are the mean and variance, respectively. In reality, the user's experienced time cost on route $r$ as days pass is regarded as multiple samples of the random travel time. Combing equation (1) with (2), the updating of mean $\tau_{k, r}^{d}$ and variance $\sigma_{k, r}^{d}$ can be formulated as

$$
\begin{aligned}
\tau_{k, r}^{d+1} & =\frac{\left(\tau_{k, r}^{d} \times d+T_{k, r}^{d}\right)}{(d+1)}, \\
\left(\sigma_{k, r}^{d+1}\right)^{2} & =\frac{\left[\left(\sigma_{k, r}^{d}\right)^{2} \times d+\left(T_{k, r}^{d}-\tau_{k, r}^{d}\right)^{2}\right]}{(d+1)},
\end{aligned}
$$

where for the initial value, i.e., when $d=1$, the mean and variance values taken are routes' free travel time.

In particular, the perceived travel time and not the real travel time is taken as a sample datum. For each user, the departure time and route choice simultaneously form a self-decided course of action that grants users the authority and responsibility to make their own decisions and implement them. So, a perceived travel time that takes into account the user's individual preference and propensity is more accurate.

3.2. Microscopic Model. Due to travel time variance, a lower cost, minimal uncertainty, and a higher probability of ontime arrival are every user's fundamental aim for day-to-day travel behaviour. The proposed microscopic model consists of each user's decision process divided into three stages.

3.2.1. Departure Time Selection. For simplicity, each user's work starting time and selected departure time on day $d+1$ are denoted as $T_{A}^{d+1}, T_{S}^{d+1}=k \times \Delta t$, respectively, where $k$ is the corresponding time slot number and $\Delta t$ is the duration of each slot. The reserved travel time for the whole journey is written as $\mathrm{bt}_{k}^{d+1}=T_{A}^{d+1}-T_{S}^{d+1}\left(\forall r \in R_{w}\right)$. Let $t_{\mathrm{ea}}, t_{\mathrm{la}}$ be acceptable early and late arrival times; in other words, a travel time $t_{k, r}^{d+1}$ that lies in the closed interval $\left[\mathrm{bt}_{k}^{d+1}-t_{\mathrm{ea}}, \mathrm{bt}_{k}^{d+1}+\right.$ $t_{\mathrm{la}}$ ] at departure slot $k$ on route $r$ is deemed as arriving punctually. However, owing to the stochastic processes of the system, uncertainties also exist in punctual arrival, so the arrival probability at slot $k$ on day $d$ can be expressed as

$$
\begin{aligned}
Z_{1}(k, d+1)= & P\left(\mathrm{bt}_{k}^{d+1}-t_{\mathrm{ea}} \leq t_{k, r}^{d+1} \leq \mathrm{bt}_{k}^{d+1}+t_{\mathrm{la}}\right) \\
= & \Phi\left(\frac{\mathrm{bt}_{k}^{d+1}+t_{\mathrm{la}}-\tau_{k}^{d+1}}{\sigma_{k}^{d+1}}\right) \\
& -\Phi\left(\frac{\mathrm{bt}_{k}^{d+1}+t_{\mathrm{le}}-\tau_{k}^{d+1}}{\sigma_{k}^{d+1}}\right) \\
\tau_{k}^{d+1}= & \frac{\sum_{\forall r} \tau_{k, r}^{d+1}}{N_{w}} \\
\sigma_{k}^{d+1}= & \frac{\sum_{\forall r} \sigma_{k, r}^{d+1}}{N_{w}} .
\end{aligned}
$$

In equation (4), $\Phi(*)$ is the standard normal distribution function. Equation (4) shows that the mean and variance of the travel time at departure slot $k$ have an average value for all paths. When users select the departure time before route choice, they will evaluate all paths prospects comprehensively.

Apart from pursuing the highest probability of punctual arrival, a more significant issue is to avoid "being late," which is assigned a high penalty. Due to the stochastic process, users pursue the smallest probability of being late:

$$
\begin{aligned}
Z_{2}(k, d+1) & =P\left(t_{k, r}^{d+1} \geq \mathrm{bt}_{k}^{d+1}+t_{\mathrm{la}}\right) \\
& =1-\Phi\left(\frac{\mathrm{bt}_{k}^{d+1}+t_{\mathrm{la}}-\tau_{k}^{d+1}}{\sigma_{k}^{d+1}}\right) .
\end{aligned}
$$

Furthermore, equations (4) and (6) assume that punctual arrival is set within a closed interval, but in reality, if a smaller delay time $t_{l}$ is strictly disallowed, then the scheduled time $t_{k, r}^{d+1}$ can be shortened to $\mathrm{bt}_{k, r}^{d+1}$. 
3.2.2. Path Choice Based on Experienced Information. For the consideration of the network's uncertainties and the users' risk propensity, the cumulative prospect theory is applied to describe users' route choice behaviour [28]. The three reference points are set as the budget time
$\mathrm{bt}_{k}^{d+1}=T_{A}^{d+1}-k \times \Delta t$, and the earliest and latest time permitted $\mathrm{bt}_{k}^{d+1}-t_{\mathrm{ea}}, \mathrm{bt}_{k}^{d+1}+t_{\mathrm{la}}$ separately. The value function $v\left(T_{k, r}^{d+1}\right)$ can be written as a piecewise function:

$$
v\left(T_{k, r}^{d+1}\right)= \begin{cases}-\lambda_{1}\left(\mathrm{bt}_{k}^{d+1}-t_{e a}-T_{k, r}^{d+1}\right)^{\alpha_{1}} ; & T_{k, r}^{d+1} \in\left(0, \mathrm{bt}_{k}^{d+1}-t_{\mathrm{ea}}\right], \\ -\lambda_{2}\left[T_{k, r}^{d+1}-\left(\mathrm{bt}_{k}^{d+1}-t_{\mathrm{ea}}\right)\right]^{\alpha_{2}} ; & T_{k, r}^{d+1} \in\left(\mathrm{bt}_{k}^{d+1}-t_{\mathrm{ea}}, \mathrm{bt}_{k}^{d+1}\right], \\ -\lambda_{3}\left(\mathrm{bt}_{k}^{d+1}+t_{\mathrm{la}}-T_{k, r}^{d+1}\right)^{\alpha_{3}} ; & T_{k, r}^{d+1} \in\left(\mathrm{bt}_{k}^{d+1}, \mathrm{bt}_{k}^{d+1}+t_{\mathrm{la}}\right], \\ -\lambda_{4}\left[T_{k, r}^{d+1}-\left(\mathrm{bt}_{k}^{d+1}+t_{l}\right)\right]^{\alpha_{4}} ; & T_{k, r}^{d+1} \in\left(\mathrm{bt}_{k}^{d+1}+t_{\mathrm{la}},+\infty\right] .\end{cases}
$$

The weighting function is

$$
\pi\left(P_{i}\right)=e^{\left[\left(-\ln P_{i}\right)^{\gamma}\right]} ; \quad i=1,2,3,4 .
$$

The path prospect value is

$$
\mathrm{PS}_{k, r}^{d+1}=\sum_{i=1, \ldots, 4} v\left(T_{k, r}^{d+1}\right) \pi\left(P_{i}\right)
$$

In the above equations, $\alpha_{i}, \lambda_{i}(i=1,2,3,4)$ are the parameters expressing users' various preferences; $P_{i}$ is the corresponding probability of $T_{k, r}^{d+1}$ within each interval. From equation (7), we can see that $v\left(T_{k, r}^{d+1}\right)$ values are positive in interval $\left(\mathrm{bt}_{k}^{d+1}-t_{\mathrm{ea}}, \mathrm{bt}_{k}^{d+1}+t_{\mathrm{la}}\right)$ but negative in others.

3.2.3. Influence of Navigation Applications. When users select their departure slot $k^{*}$ through the procedure explained in (1), simultaneously, the route $r^{*}$ with the largest prospect in assumption (2) is determined in mind. We can call route " $r *$ " the "preselected route." The choice before departure is determined by the perceived travel time, punctual reliability, and risk avoidance, which are the most important issues considered. Therefore, the departure time is selected based on experience information, and a "preselected route" is determined along with it.

However, when users start a journey at time slot $k$, the real-time information from navigation will be the actual time cost in a certain degree, which is different from the assumption commonly encountered in the literature, as navigation information does not cover all the paths. The recommended best route will be an important influence for route changing. The reliance on the "preselected route," namely, experience inertia, and the performance of recommended alternatives are also been taken into consideration. The details and decision stages of the process are as follows:

(1) If the "preselected route" is suffering unexpected congestion and its travel time exceeds the reserved time, i.e., $t_{k, r}^{d+1} \geq \mathrm{bt}_{k}^{d+1}$, users will change their initial plan to avoid being late, and the alternative which has the highest utility will be chosen.
(2) If the "preselected route" performs normally, but based on real-time information, a better alternative $r^{\prime}$ exists, or the difference $t_{k, r}^{d+1} \geq \mathrm{bt}_{k, r^{\prime}}^{d+1}$ is larger than a fixed tolerance describing the experience inertia, and users will adjust their decision. Otherwise, users will maintain their original route.

\section{Deriving the Aggregated Model}

4.1. Aggregated Model for Preselection. As discussed in 3.2, on day $(d+1)$, users will select a route $r$ and a departure slot $k$ to ensure achievement of the double objective described by equations (4) and (6). For this biobjective problem, a simple method is presented, which adds a weighting coefficient to transform it into a single objective problem.

$$
Z(k, d)=Z_{1}(k, d)-\theta Z_{2}(k, d)
$$

Since users have a lower tolerance for lateness, in equation (10), $\theta>1$. Certainly, a more accurate and complicated solution is possible to define the relationship between punctual arrival and lateness avoidance. Some cases where $Z(k, d)$ could have a negative value have no influence on the flow revision ratio because the absolute value of $Z(k, d)$ is the core element.

To derive the aggregated stochastic model, we need to make an explicit description of how the route flow $X_{k, r}^{d+1}\left(r \in R_{w}\right)$ or link flow $X_{k, a}^{d+1}(a \in A)$ change over consecutive days. In Section 3, we explained the individual's behaviour, but for a real network, the common phenomenon is that two or more users can simultaneously revise their choice on the same day or the same departure slot. In the aggregated model, the adjustment process discussed in Section 3 is not a certain result because a personal revision must take others' decisions into consideration, which will result in network flow fluctuation. Therefore, for a group of users, route and departure time choice revision occur at a certain probability.

According to the transformation model proposed by Smith [31], which assumed users are bounded rational and rely on experience, an aggregate dynamic transformation 
model considering route and departure time revision simultaneously is used to describe spatial and temporal flow.

4.1.1. Flow Revision between Departure Slot. First, following Smith's approach to define the revised flow before departure using the alternatives $k_{1}, k_{2} \in K / k$, the flow $X_{k}^{d+1}$ on slot $k$ of day $(d+1)$ can be written as

$$
X_{k}^{d+1}=X_{k}^{d}+\sum_{\forall k_{1} \in(K / k)} X_{k_{1}}^{d} \longrightarrow k\left(X_{k_{1}}^{d}\right)-\sum_{\forall k_{2} \in(K / k)} X_{k_{2}}^{d} \longrightarrow k\left(X_{k}^{d}\right) .
$$

The aggregate inflow from slot $k_{1}$ is

$$
X_{k_{1}}^{d} \longrightarrow k\left(X_{k_{1}}^{d}\right)=\delta_{1} \cdot \frac{\max \left\{Z(k, d)-Z\left(k_{1}, d\right)-\eta_{1}, 0\right\}}{\left|\left(k-k_{1}\right) \cdot \Delta t\right|} \cdot X_{k_{1}}^{d} .
$$

The aggregate outflow to slot $k_{2}$ is

$$
\begin{aligned}
X_{k_{2} \longrightarrow k}^{d}\left(X_{k}^{d}\right) & =\delta_{1} \cdot \frac{\max \left\{Z\left(k_{2}, d\right)-Z(k, d)-\eta_{1}, 0\right\}}{\left|\left(k-k_{2}\right) \cdot \Delta t\right|} \cdot X_{k}^{d}, \\
X_{k}^{d} & =\sum_{\forall r \in R_{w}} X_{k, r}^{d},
\end{aligned}
$$

where $X_{k, r}^{d}$ represents the flow on path $r$ at interval $k$ on day $d$. The above equations can be explained as follows: the revision coefficient is denoted as $\delta_{1}$ to describe the probability of adjustment; $\max \left\{Z(k, d)-Z\left(k_{1}, d\right)-\eta_{1}, 0\right\}$ defines that the conversion probability is in positive proportion to the difference between $Z(k, d)-Z\left(k_{1}, d\right)$ and the punctuality threshold $\eta_{1}$; otherwise, $|-k \cdot \Delta t|$ expresses an inverse relationship because of the users' resistance to earlier departure.

4.1.2. Flow Revision between Routes. The constraints of flow conservation are

$$
X_{k}^{d+1}=\sum_{\forall r \in R_{w}} X_{k, r}^{d+1}
$$

The flow revision between routes is therefore

$$
X_{k, r}^{d+1}=X_{k, r}^{d}+\sum_{\forall r_{1} \in R_{w} / r} X_{k, r_{1}}^{d} \longrightarrow r-\sum_{\forall r_{2} \in R_{w} / r} X_{k, r}^{d} \longrightarrow r_{2} .
$$

The aggregate inflow from route $r_{1}$ now becomes

$$
X_{k, r_{1}}^{d} \longrightarrow r\left(X_{k, r_{1}}^{d}\right)=\delta_{2} \times \max \left\{\mathrm{PS}_{k, r_{1}}^{d}-\mathrm{PS}_{k, r}^{d}-\eta_{2}, 0\right\} \times X_{k, r_{1}}^{d} \text {, }
$$

while the aggregate outflow to route $r_{2}$ is

$$
X_{k, r}^{d} \longrightarrow r_{2}\left(X_{k, r}^{d}\right)=\delta_{2} \times \max \left\{\mathrm{PS}_{k, r_{2}}^{d}-\mathrm{PS}_{k, r}^{d}-\eta_{2}, 0\right\} \times X_{k, r}^{d} .
$$

In the above equations, we denote the route prospect threshold as $\eta_{2}$, and $\delta_{2}$ is the route revision coefficient. To deduce the vector forms of the above model, equations (11) (17) can be rewritten as follows

Revision flows between departure slots

$$
S_{1}\left(X_{\text {time }}^{d}\right)=\sum_{\forall k_{1}, k_{2}} \delta_{1} \times \frac{\max \left\{Z(k, d)-Z\left(k_{1}, d\right)-\eta_{1}, 0\right\}}{\left|\left(k-k_{1}\right) \cdot \Delta t\right|} \times X_{\text {time }}^{d} \times \Psi_{k_{1}, k_{2}} .
$$

Vector form for all departure slots is

$$
X_{\text {time }}^{d+1}=X_{\text {time }}^{d}+S_{1}\left(X_{\text {time }}^{d}\right) \text {. }
$$

Revision flows between routes at slot $k$

$$
S_{2}\left(X_{k, \text { route }}^{d}\right)=\sum_{\forall r_{1}, r_{2}} \delta_{2} \times \max \left\{\left(\mathrm{PS}_{k, r_{1}}^{d}-\mathrm{PS}_{k, r_{2}}^{d}-\eta_{2}, 0\right)\right\} \times X_{k, \text { route }}^{d} \times \Psi_{r_{1}, r_{2}} \text {. }
$$

Vector form at all routes is

$$
X_{k, \text { route }}^{d+1}=X_{k, \text { route }}^{d}+S_{2}\left(X_{k, \text { route }}^{d}\right)
$$

where, $\Psi_{k_{1}, k_{2}}$ is called the indicating vector, in which row $k_{1}$ equals -1 , row $k_{2}$ values are equal to 1 , and other rows are set zero. $\Psi_{r_{1}, r_{2}}$ is defined in a similar manner to $\Psi_{k_{1}, k_{2}}$.

4.2. Rerouting with Navigation Guidance. As in the above description, users will change their initial choice with navigation guidance assistance. When they start their journey at a preselected departure time, users compare their perceived route with the two recommended alternatives; if some unexpected enroute congestion is present or a shorter path is found, some revision is necessary and available. Noticeably, different from taking the prospect value for criteria for preselection, rerouting choice will compare realtime travel cost at departure slot for the preselected and recommended paths. This setting can be explained as follows: for travel plans before departure, choice is based on the perceived cost and experience, so it is obvious that users examine their perceived information's variability and 
randomness. However, when starting their journey, users show complete trust on the real-time information of preselected and the navigation system-recommended paths.
In the same manner, the route conversion probability and flow should satisfy the following constraints and conditions:

$$
\begin{aligned}
& \sum_{\forall r \in R_{w}} X_{k, r}^{d+1}=\sum_{\forall r \in R_{w}} \overline{X_{k, r}^{d+1}} \\
& \overline{X_{k, r}^{d+1}}=X_{k, r}^{d+1}+\sum_{\forall r_{1} \in R_{w} / r} \overline{X_{k, r_{1}}^{d+1} \longrightarrow r}-\sum_{\forall r_{2} \in R_{w} / r} \overline{X_{k, r}^{d+1} \longrightarrow r_{2}}, \\
& \overline{X_{k, r^{\prime}}^{d+1} \longrightarrow r}= \begin{cases}\delta_{3} \times \max \left\{\overline{t_{k, r^{\prime}}^{d+1}}-\overline{t_{k, r}^{d+1}}-\eta_{3}, 0\right\} \times X_{k, r^{\prime}}^{d+1} ; & b t_{k}^{d+1}>\overline{t_{k, r^{\prime}}^{d+1}} \\
\delta_{3} \times \max \left\{\overline{t_{k, r^{\prime}}^{d+1}}-\overline{t_{k, r}^{d+1}}, 0\right\} \times X_{k, r^{\prime}}^{d+1} ; & b t_{k}^{d+1} \leq \overline{t_{k, r^{\prime}}^{d+1}}\end{cases}
\end{aligned}
$$

Equation (24) is based on the adjustment rules mentioned above. In these equations, $X_{k, r}^{d+1}$ is the real-time flow on route $r$ at slot $k, \overline{X_{k, r^{\prime}}^{d+1} \longrightarrow r}$ denotes the allowed outflow revision from route $r^{\prime}$ to $r$, and the real-time travel time is expressed as $\overline{t_{k, r}^{d+1}} . \delta_{3}$ and $\eta_{3}$ are the parameters used to describe users' risk propensity.

\section{Theoretical Properties of the Proposed Model}

In this section, we discuss the theoretical properties of the equilibrium's stability. The existence and the stability of the equilibrium in the day-to-day and within-day dynamic processes are also analyzed.
Now, we rewrite (18) and (21) as follows:

$$
X_{k}^{d+1}=X_{k}^{d}+F_{1}\left(\delta_{1}, \eta_{1}, \Psi_{k_{1}, k_{2}}, T_{k, r}^{d}\right) \times X_{k}^{d} .
$$

In (4) and (6), the punctual arrival probability is dependent on $T_{k, r}^{d}$. Therefore, $F_{1}\left(\delta_{1}, \eta_{1}, \Psi_{k_{1}, k_{2}}, T_{k, r}^{d}\right)$ denotes a function with respect to parameters $\delta_{1}, \eta_{1}, \Psi_{k_{1}, k_{2}}, T_{k, r}^{d}$.

$$
X_{k, r}^{d+1}=X_{k, r}^{d}+F_{2}\left(\delta_{2}, \eta_{2}, \Psi_{r_{1}, r_{2}}, \operatorname{PS}_{k, r}^{d}\right) \times X_{k}^{d}
$$

Considering (7)-(9), we notice $\mathrm{PS}_{k, r}^{d}$ w.r.t $T_{k, r}^{d}, T_{k, r}^{d}$ w.r.t $X_{k}^{d+1}$ with the flow loading model. So, (26) can be rewritten as

$$
X_{k, r}^{d+1}=X_{k, r}^{d}+F_{2}^{\prime}\left(\delta_{1}, \delta_{2}, \eta_{1}, \eta_{2}, \lambda_{j}, \Psi_{k_{1}, k_{2}}, \Psi_{r_{1}, r_{2}}, T_{k, r}^{d}\right) \times X_{k}^{d}, \quad j=1,2,3,4
$$

Theorem 1 (Inexistence). The existence of the equilibrium solution formulated by (25) and (27) is not ensured due to the theoretical properties.

Proof. The functions $F_{1}$ and $F_{2}^{\prime}$ forming the fixed-point equations in (25) and (27) are not ensured to be continuous due to the piecewise function in (7) and the indication vectors $\Psi_{k_{1}, k_{2}}$ and $\Psi_{r_{1}, r_{2}}$. So, the domain set $\Omega_{1}$ is not continuous and convex. Since the basic conditions of Brouwer's theorem cannot be applied [32], the fixed-point solution does not exist. Similarly, in domain set $\Omega_{2}$ of the models (23) and (25) with enroute information, the inexistence of fixed equilibrium is concluded.

Theorem 2 (Oscillation equilibrium). Corresponding to the inexistence of the fixed-point equilibrium, a periodical oscillation state in a limited boundary called oscillation equilibrium can be found for the dynamic learning model.

Proof. First, the domain set $\Omega_{1}$ of the fixed-point function is the set of feasible link-departure time-flow vectors, identified by demand and travel time conservation. Obviously, the solution is positive, limited, and discrete. Second, for the dynamic process, if $\mathrm{PS}_{k, r_{i}}^{d}-\mathrm{PS}_{k, r_{j}}^{d}(j \neq i)>\eta_{2}$ in a solution $X_{k, r}^{d}$, the next day, $X_{k, r_{i}}^{d+1}$ increases by a certain range constrained to other parameters. Conversely, $X_{k, r_{i}}^{d+1}$ decreases. Third, due to one-to-one mapping relationship between $X_{k, r}^{d}$ and $\mathrm{PS}_{k, r}^{d}, Z_{k, r}^{d}$, if two identical solutions $X_{k, r}^{d}=X_{k, r}^{d^{\prime}}(\forall r, k)$, we deduce $X_{k, r}^{d+1}=X_{k, r}^{d^{\prime}+1}$. In other words, the periodic oscillation appears at the point of identical solutions. Furthermore, the limited and discrete domain set guarantees the existence of two equal solutions. Therefore, it verified periodical oscillation after a disordered learning course from the initial state.

Corollary 1. For the impact of real-time route navigation, the solution oscillation range in (23) and (24) is larger than the case which is considered without enroute information.

Proof. In models (23) and (24), a real-time flow adjustment is indicated to occur following the route preselection before departure. We denote solutions $X_{k, r}^{d_{1}, \max }{ }_{\varepsilon} \Omega_{1}, X_{k, r}^{d_{2}, \min }{ }_{\varepsilon} \Omega_{1}$, $\bar{X}_{k, r}^{d_{3}, \max }{ }_{\varepsilon} \Omega_{2}, \overline{X_{k, r}^{d_{4}, \min }} \varepsilon \Omega_{2}$ as the maximum and the minimum 
values in the domain sets $\Omega_{1}$ and $\Omega_{2}$. According to the models (16) and (17), $\exists \mathrm{PS}_{k, r_{i}}^{d_{1}^{\prime}}<\mathrm{PS}_{k, r}^{d_{1}}\left(r_{i} \neq r\right)$ at point $X_{k, r}^{\max }$; then, on the basis of (7), we conclude $\exists t_{k, r_{j}}^{d_{1}^{\prime}}>t_{k, r}^{d_{1}}$. In model (23), it means a portion of travels will choose route $r$ with the real-time information and results in $X_{k, r}^{d_{1}, \max } \leq \overline{X_{k, r}^{d_{3}, \max }}$. Similarly, at point $X_{k, r}^{d_{2}, \text { min }}, \exists \mathrm{PS}_{k, r_{i}}^{d_{2}^{\prime}}>\mathrm{PS}_{k, r}^{d_{2}}, \exists t_{k, r_{j}}^{d_{2}^{\prime}}<t_{k, r}^{d_{2}}$ leads to $X_{k, r}^{d_{1}, \min } \geq \overline{X_{k, r}^{d_{4}, \min }}$

Corollary 2. The solution oscillation range is positive related to the parameters $\eta_{1}, \eta_{2}, \delta_{1}, \delta_{2}$.

Proof. Because larger $\eta_{1}$ and $\eta_{2}$ correspond to bigger gap of path prospect and punctual probability, we conclude that $\left(X_{k, r}^{\max }-X_{k, r}^{\min }\right) \propto\left(P S_{k, r}^{\max }-P S_{k, r}^{\min }\right) \propto\left(Z_{k, r}^{\max }-Z_{k, r}^{\min }\right) \propto \eta_{1}, \eta_{2}$. The oscillation range positively correlates to $\eta_{1}$ and $\eta_{2}$. For a solution $X_{k, r}^{d}$, we denote $X_{k, r}^{d+1}\left(\delta_{1}, \delta_{2}\right)$ as flow on day $d+1$ with parameters $\delta_{1}, \delta_{2}$. If $X_{k, r}^{d+1}>X_{k, r}^{d}$, larger $\delta_{1}, \delta_{2}$ guarantees more revision flow. Conversely, if $X_{k, r}^{d+1}<X_{k, r}^{d}$, larger $\delta_{1}, \delta_{2}$ leads to less revision flow. In other words, larger $X_{k, r}^{\max }$ and smaller $X_{k, r}^{\min }$ could be ensured by larger $\delta_{1}, \delta_{2}$. Therefore, the solution oscillation range positively correlates to $\delta_{1}, \delta_{2}$.

\section{Experimental Results and Discussion}

The proposed model presents a few innovative points which differentiate our approach from previous studies. This problem has two-time axes: the axis of within-day time (denoted by interval $k$ ) and the axis of day-to-day time (denoted by $d$ ). In the within-day model, the time horizon is initially discretized into a set of equal-length time intervals denoted as $k=1,2, \ldots, K$. For each time step, the rerouting model calculates the final flow on each route, and the BPR function $t_{a}=t_{a}^{0} \times\left[1+0.15 \times\left(x_{a} / c_{a}\right)^{4}\right]$ as a flow-loading model is used to evaluate real-time travel time. The day-to-day model presented above is a flow assignment course along days, which outputs spatial-temporary flow assignment before departure. Therefore, there is a cyclic iteration relationship between the within-day and day-to-day models.

6.1. Numerical Example. In this section, some experiments are conducted to validate the model on a road network consisting of three paths between a unique origin and a destination. The departure time zone is set as $(7: 30-9: 00)$ and discretized into 9 intervals of equal duration, where each interval is $10 \mathrm{~min}$ due to precision deficiency of travellers' habit on time choice and to avoid excessively long computational times. A total of 1000 vehicles in each departure slot are travelling between this origin-destination pair everyday during the morning peak hours. Drivers select their departure time and route at the origin so as to maximize their utility. On the first day, for the initial flow of this model, we simply set the free travel time $\sum_{a \in r} t_{a}^{0}$ as $t_{k, r}^{1}$ and $\tau_{k, r}^{1}$, while $\sigma_{k, r}^{1}=\varphi \cdot t_{k, r}^{1}$, and coefficient $\varphi=0.2$ can be estimated as the real travel time's variance range, acquired from statistical information of paths in the same region. The stochastic choice parameters for departure time and route choice are denoted as $\theta_{P}=\theta_{T}=0.3$ for the first day. Unless otherwise specified, the values of key parameters are those reported in Table 1.

6.2. Spatial-Temporary Flow. The purpose of this experiment was to validate the dynamic route choice model and investigate the effects of navigation on spatial-temporary flow through a comparison of navigation-considering and nonconsidering models which involve only decisions before departure and eliminates real-time rerouting options. The time horizon was set as 200 days, and the routes' spatialtemporary flow at each slot in within-day along the 200 days demonstrates the day-to-day dynamic evolution and the final equilibrium states.

Figure 1 is composed of 6 plots which show the three paths' flows produced in this instance. Two plots in the same row show the spatial-temporary flow difference on two same routes and navigation performance obtained through different model settings. Meanwhile, plots in the same column represent results of all three paths for a certain mode. For convenience, we call the model without navigation as circumstance I and the other as circumstance II.

The six plots suggest some common and specific phenomena for the two models: (1) all flow curves converge to an equilibrium state after a period of oscillation. Unfortunately, due to the three-dimensional complexity, the path flow datum at each interval cannot be checked clearly. However, the oscillation process and final stability which are more important can be observed. However, it is worth noting that the equilibrium is somewhat different in each case. In circumstance I, the curve reaches an approximate fixed state at $d=150$, route flow at each departure time slot approaches to static, and oscillation range is smaller than 20 vehicles. In contrast, in circumstance II, we observe an obvious oscillation equilibrium with a cycle period of 5 days after $d=27$. The phenomenon implies that travellers show more utility sensitivity and are more dependent on and confident of their navigation system than experience. The conclusion coincides with Corollary 1. (2) In two circumstances, all the plots suggest that the flow between departure time $(8: 00,8: 30)(k=4,5,6,7)$ is apparently larger and that although the travel time is longer, the prospect is larger. This finding suggests that departing at an appropriate time which affords a high on-time probability is more important and attractive than suffering an increased travel duration cost.

Figures 2 and 3 also illustrate the above conclusions. These two plots present the value of $\sum_{\forall k \in K} X_{k, r}^{d+1}$ and $\sum_{\forall r \in R_{w}} X_{k, r}^{d+1}$, respectively, in the two circumstances along the time horizon in order to determine the features of route and departure time flows. The curves also indicate an approximate fixed state and oscillation equilibrium for different model circumstances, similar to Figure 1. This suggests that the status of stability or instability is highly dependent on information and user reliance.

Specifically, from the proposed model, we see that any state that satisfied $Z\left(k_{1}\right)-Z\left(k_{2}\right) \leq \eta_{1}\left(\forall k_{1}, k_{2} \in K\right)$ and $\mathrm{PS}_{k, r_{1}}^{d}-\mathrm{PS}_{k, r_{2}}^{d} \leq \eta_{2}\left(\forall r_{1}, r_{2} \in R_{w}\right)$, i.e., utility difference is within tolerance, flow revision did not occur and the 
TABle 1: Default parameter values.

\begin{tabular}{lcc}
\hline Notation & Description & Value \\
\hline$T_{A}$ & Work started time & $9: 00$ \\
$t_{a}^{0}$ & Free travel time & $(30,25,35) \mathrm{min}$ \\
$K$ & Intervals number & 9 \\
$c_{a}$ & Link capacity & $(250,200,300) \mathrm{veh} / \mathrm{min}$ \\
$t_{\mathrm{ea}}$ & Permitted earlier time & $0.1 \mathrm{bt}_{k}^{d+1}$ \\
$t_{\mathrm{la}}$ & Permitted later time & $0.05 \mathrm{bt}_{k}^{d+1}$ \\
$\mathrm{~L}$ & The reserve memory days & 4 \\
$\theta_{P}=\theta_{T}$ & Validation parameters & 0.3 \\
$\eta_{1}$ & Punctual arrival tolerance & 0.4 \\
$\eta_{2}$ & Path prospect tolerance & 0.4 \\
$\eta_{3}$ & Real-time difference tolerance & 0.4 \\
$\delta_{i}$ & Revision ratio & 0.5 \\
$d$ & Total demand at each slot & $1000 \mathrm{veh} / \mathrm{m}$ \\
$\alpha_{i}, \lambda_{i}, \gamma$ & & $\alpha_{i}=0.88(i=1,2,3,4)$ \\
& Parameters in prospect function & $\lambda_{i}=[0.2,0.3,0.3,0.5]$ \\
$y_{d-l}$ & & $\gamma=0.74$ \\
\hline
\end{tabular}

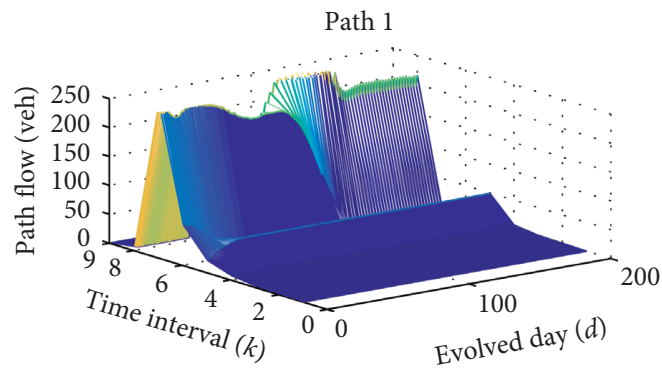

Path 2

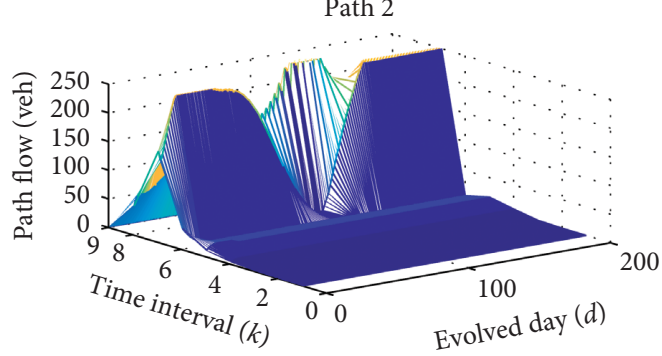

Path 3

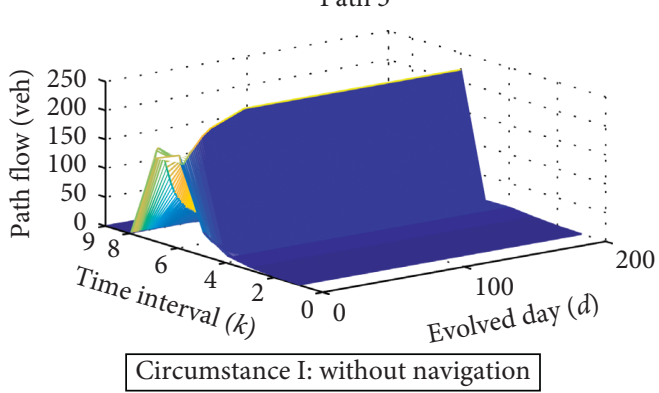

(a)

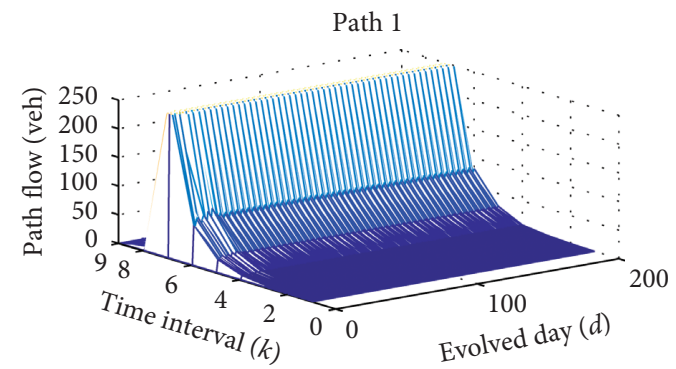

Path 2

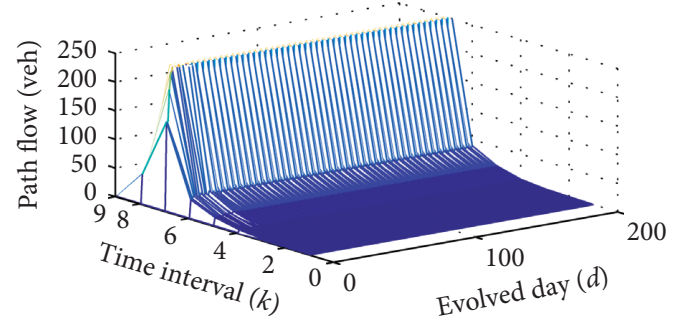

Path 3

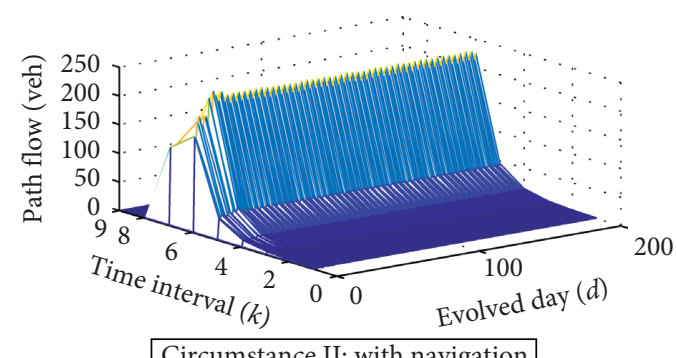

Circumstance II: with navigation

(b)

Figure 1: Flows on each path at each time interval. (a) Circumstance I: without navigation. (b) Circumstance II: without navigation. 


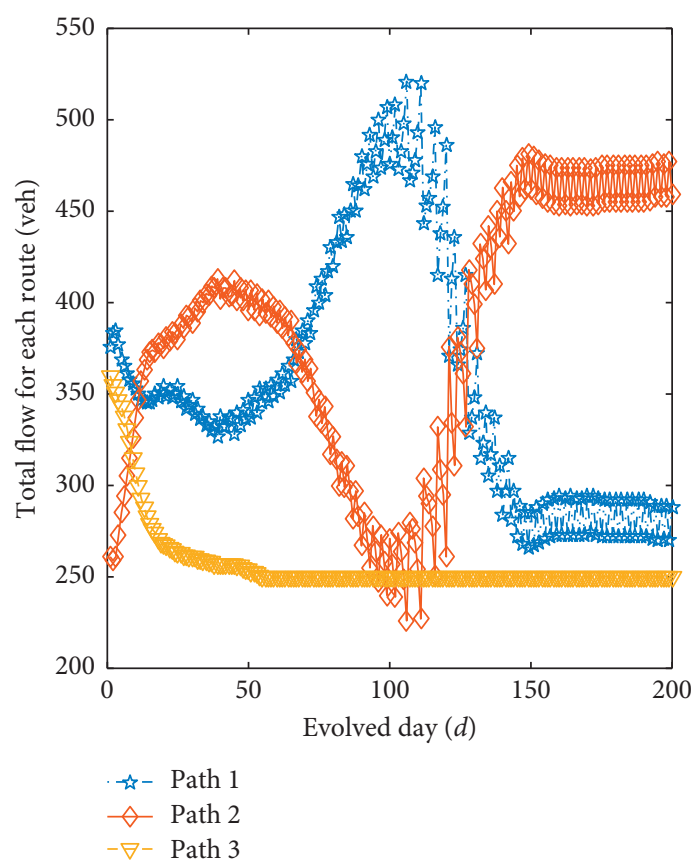

(a)

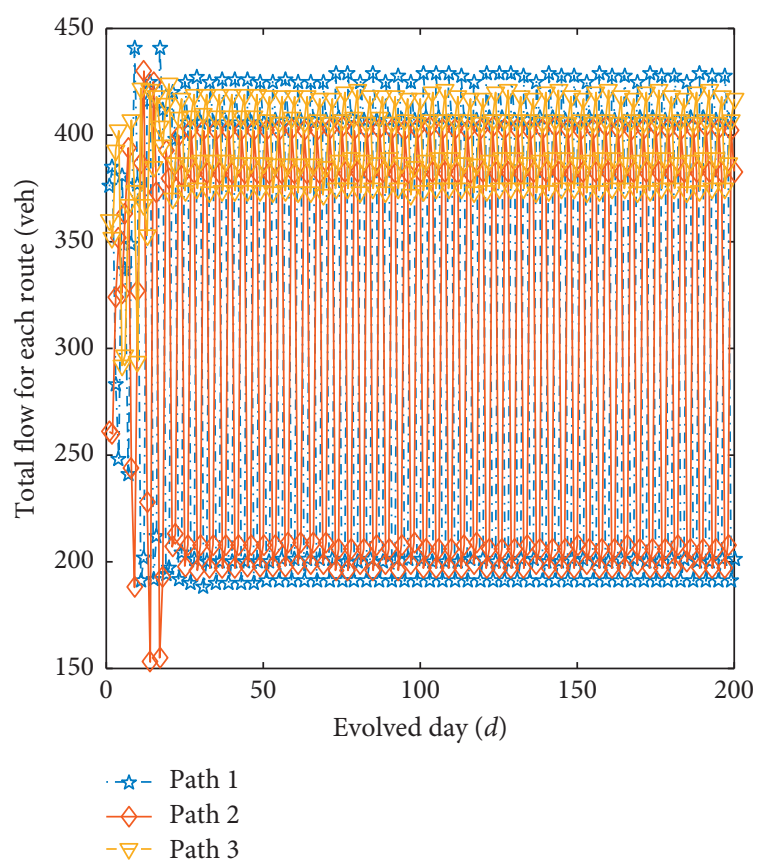

(b)

FIgURE 2: Total route flow in two circumstances.

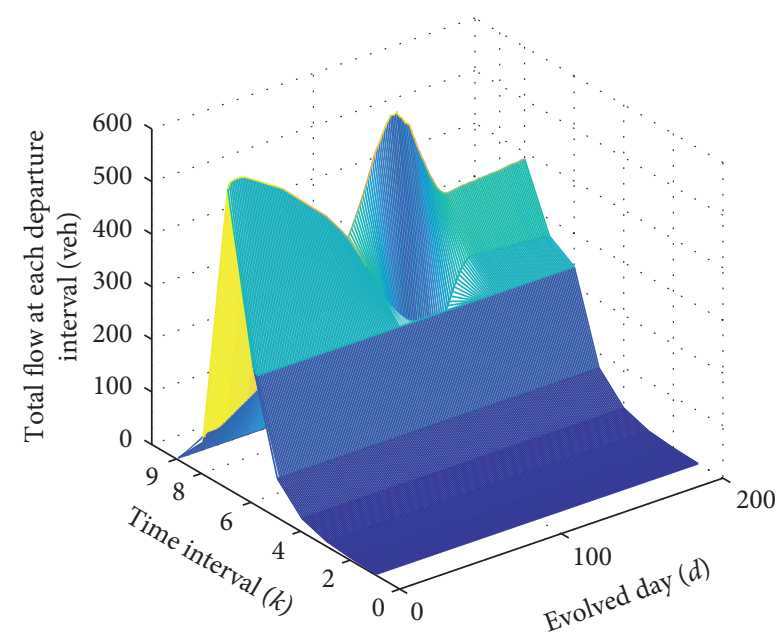

(a)

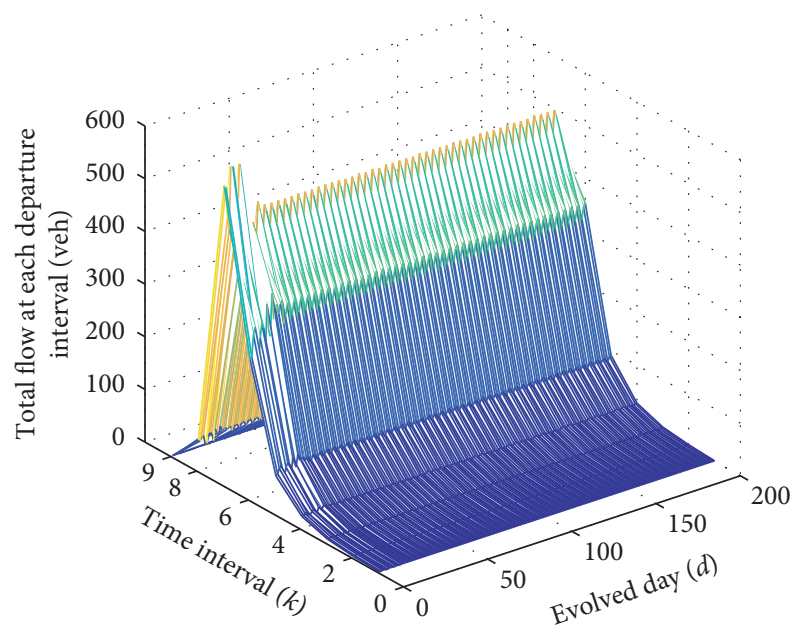

(b)

Figure 3: The total departure time flow in two circumstances.

network eventually reached stability. This implies that the day-to-day traffic system's final state is not unique and diversified due to the variability of the initial states.

Obviously, the initial setting above is reasonable for a newly built network. However, for a network in service, in order to analyze the process of stability-oscillation, stability due to unpredictable disruptions, for instance a capacity drop or rise from day $d$, real experience before day $d$ is a reasonable choice of initial conditions.
6.3. Comparison with UE. In the proposed model, due to the travellers' bounded rationality when considering day-to-day route and navigation information, the final states (after some oscillations) were not consistent with UE in most instances. When the system reached stability, the prospect value on the chosen paths at each time slot will be identical and the constraints in the following UE model will be satisfied. However, from the results illustrated in Figures 1-3, we see that $\mathrm{PS}_{k, r}^{200} \neq \mathrm{PS}_{k^{\prime}, r^{\prime}}^{200}\left(\forall k, k^{\prime} \in K, \forall r, r^{\prime} \in R_{w}\right)$. Obviously, user 
(1) Input: all the variables in Table 1; total path number $R$;

(2) Output: flow variables $X_{k, r}, X_{k}$.

(3) Initialization: for each departure interval $k$, divide demand $D$ into $K$ segments, set $X_{k}^{0}=D / K,(1 \leq k \leq K)$. Denote $m$ as outer iteration index and initial value $m=0$. Denote $n$ as the inner iteration index and initial value $n=0$.

(4) Main loop.

(5) Inner iteration: path flow assignment.

(6) xFor $1 \leq k \leq K$, denote $\mathrm{PS}_{k, r}^{0}$ as the initial prospect, calculated using equations (7)-(9).

(7) When $n=1,2, P_{k, r}^{n}=\left(\exp \left(-\theta_{P} \times P S_{k, r}^{n-1}\right) / \sum_{\forall r \in R_{w}} \exp \left(-\theta_{P} \times P S_{k, r}^{n-1}\right)\right), X_{k, r}^{n}=X_{k}^{m} \times P_{k, r}^{n}$; then update PS $S_{k, r}^{n}$, using equations (7)-(9); the $\theta_{P}$ is stochastic parameter for route choice;

(8) If $\left|X_{k, r}^{n}-X_{k, r}^{n-1}\right| / X_{k, r}^{n-1}>\varepsilon_{1}$, do: $n=n+1$, method of successive averages (MSA) to update flow $P_{k, r}^{n}=\left(\exp \left(-\theta_{P} \times \mathrm{PS}_{k, r}^{n-1}\right) / \sum_{\forall r \in R_{w}} \exp \left(-\theta_{P} \times \mathrm{PS}_{k, r}^{n-1}\right)\right) F_{k, r}^{n}=X_{k}^{m} \times P_{k, r}^{n}, X_{k, r}^{n+1}=X_{k, r}^{n}+\left(F_{k, r}^{n}-X_{k, r}^{n}\right) / n$

(9) If $\left|X_{k, r}^{n}-X_{k, r}^{n-1}\right| / X_{k, r}^{n-1} \leq \varepsilon_{1}(n \geq 3)$, exit the inner iteration.

(10) Outer iteration: departure time assignment.

(11) $m=m+1$. For $1 \leq k \leq K$, set $\mathrm{PS}_{k}^{m}=\sum_{\forall r \in R_{w}} \mathrm{PS}_{k, r}^{n} / R, P_{k, r}^{n}=\left(\exp \left(-\theta_{P} \times \mathrm{PS}_{k, r}^{n-1}\right) / \sum_{\forall r \in R_{w}} \exp \left(-\theta_{P} \times \mathrm{PS}_{k, r}^{n-1}\right)\right) . F_{k}^{m}=D \times P_{k}^{m}, \theta_{T}$ is the stochastic parameter for time choice.

(12) When $m=1, X_{k}^{m}=F_{k}^{m}$.

(13) When $m \geq 2$, if $\left|X_{k, r}^{n}-X_{k, r}^{n-1}\right| / X_{k, r}^{n-1}>\varepsilon_{1}$, do: MSA to update flow: $X_{k}^{m}=X_{k}^{m-1}+\left(X_{k}^{m-1}-F_{k}^{m}\right) / m$. Output: $X_{k}^{m}$, enter into the inner iteration.

(14) If $\left|X_{k, r}^{n}-X_{k, r}^{n-1}\right| / X_{k, r}^{n-1} \leq \varepsilon_{1}$, end.

Algorithm 1: RTCIA for the UE model.

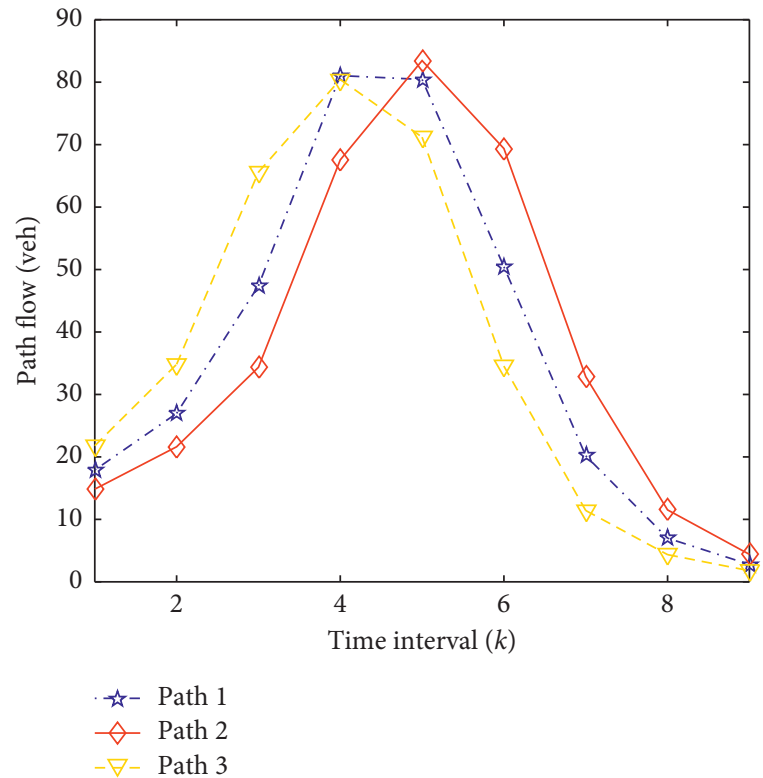

(a)

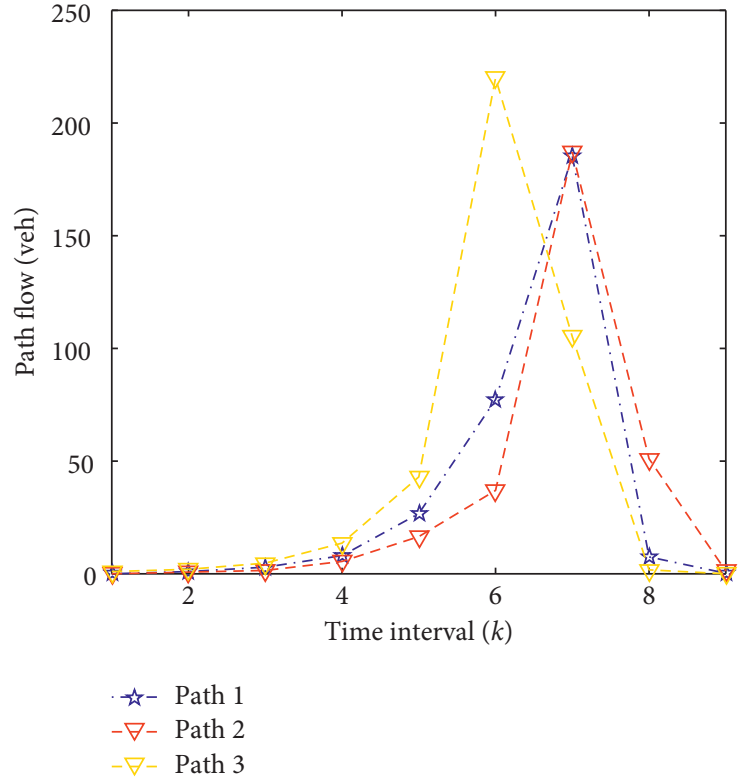

(b)

Figure 4: Comparison of two kinds of equilibria. (a) UE equilibrium. (b) Day-to-day equilibrium.

equilibrium did not agree with these results. Therefore, in this section, we construct a route-time cross-iteration algorithm (RTCIA) to solve the UE model with route and departure time choice simultaneously, and a comparison is performed to search differences between the proposed model and the UE model.

The UE model can be formulated as

$$
\begin{aligned}
& X_{k, r} \cdot\left(\mathrm{PS}_{k, r}-\mathrm{PS}_{k, r}^{*}\right)=0, \\
& \mathrm{PS}_{k, r}-\mathrm{PS}_{k, r}^{*} \geq 0, \\
& X_{k}=\sum_{\forall r \in R_{w}} X_{k, r}, X_{r}=\sum_{\forall k \in K} X_{k, r}, D=\sum_{\forall k \in K} \sum_{\forall r \in R_{w}} X_{k, r} .
\end{aligned}
$$




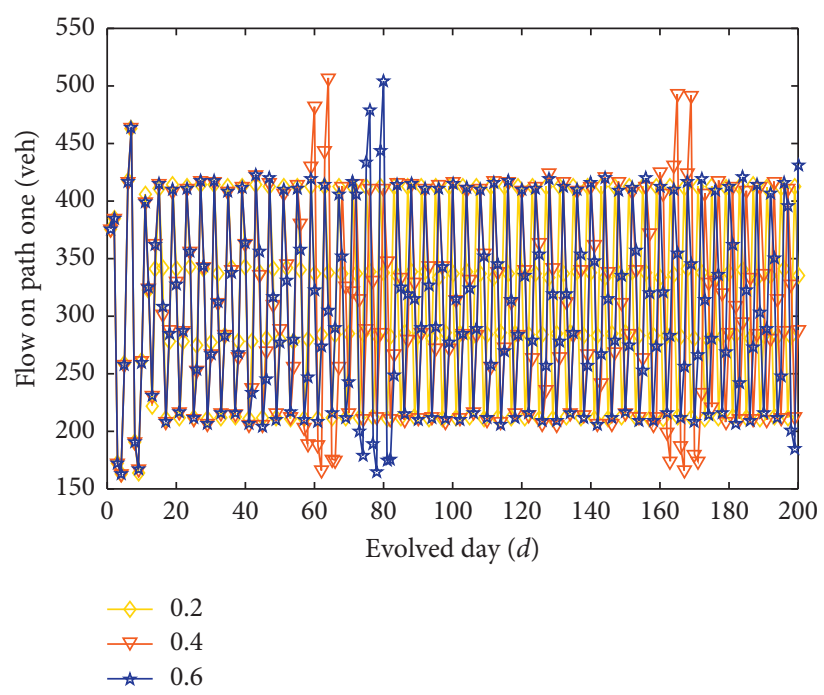

Figure 5: Results of path flows for different values of $\eta_{i}$.

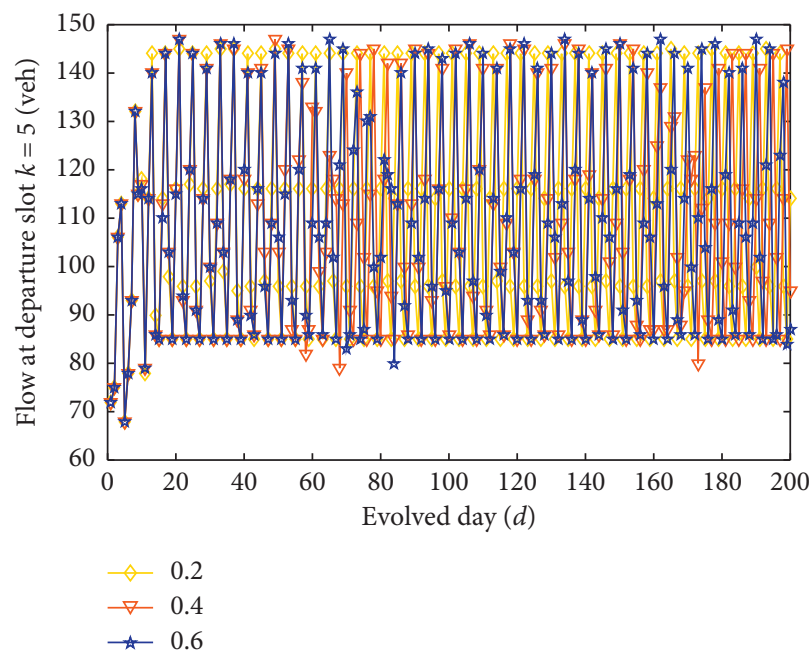

FIgURE 6: Results of time flow for different values of $\eta_{i}$.

If $\mathrm{PS}_{k, r}>\mathrm{PS}_{k, r}^{*}, X_{k, r}=0$, it is identical with Wardrop's equilibrium definition. $X_{k}$ and $X_{r}$ represent the sum values of all paths and all routes, respectively.

The route-time cross-iteration algorithm (RTCIA) is constituted by two crossed flow assignment processes. One iteration is the path flow $\left(X_{r}\right)$ equilibrium assignment with the total flow $X_{k}$ at time interval $k=t / \Delta t$, while the other is to calculate each slot flow $X_{r}$ to reach the departure time equilibrium.

The RTCIA for the UE model is described as the pseudocode for Algorithm 1.

In order to compare the results of the day-to-day model with the UE model appropriately, RTCIA was applied on the example in Section 6.3. All the parameters in RTCIA were given the same value as the day-to-day model aiming to ensure a fair comparison. Figure 4 illustrates the three paths' flow at the steady state (considering the oscillation equilibria and taking the sum route flow $\sum_{k=160}^{200} X_{k, r}^{n+1} / 40$ as the stable flow in the day-to-day model). In the left plot, $\mathrm{PS}_{k, r}^{200} \approx \mathrm{PS}_{k^{\prime}, r^{\prime}}^{200}$ $\left(\forall k, k^{\prime} \in K, \forall r, r^{\prime} \in R_{w}\right.$ ), which suggests a user equilibrium within accepted errors. It is clear that the two plots have considerable differences. For UE, the flow at each interval varies more gently (5 83 veh) than in the day-to-day model (0 220 veh). However, the results are all reasonable. First, with the impact of tolerance parameters $\eta_{1}, \eta_{2}$, the boundedrational model is unable to reach a UE equilibrium. Second, the travels are concentrated mainly in the peak time to guarantee a higher punctual probability. This indicates that 


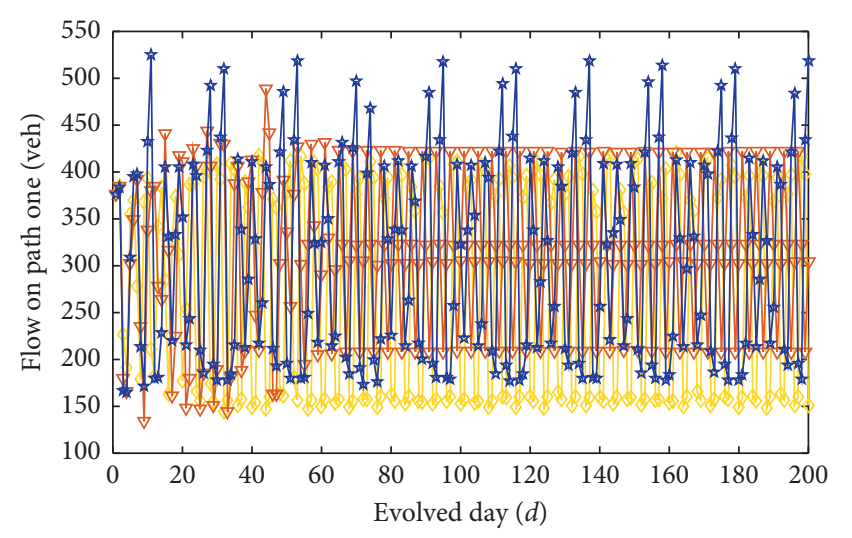

$\smile \quad 0.2$
$\neg \quad 0.4$
$-\quad 0.6$

Figure 7: Results of path flows for different values of $\delta_{i}$.

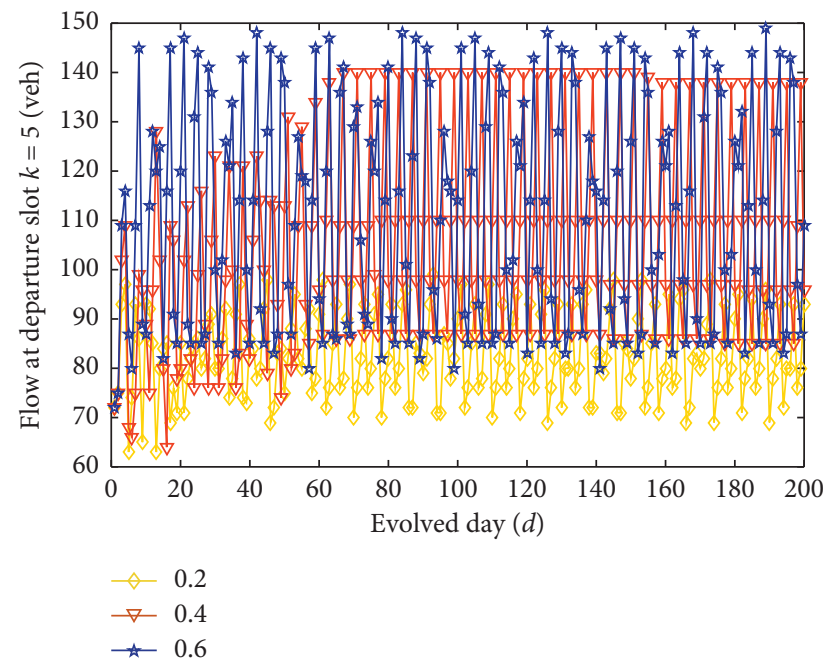

Figure 8: Results of time flow for different values of $\delta_{i}$.

the degree of difference among users' travel cost in the various models is highly dependent on travellers' decision psychology and acceptance of the utility gap.

6.4. Parameter Analysis. In the proposed model and example, many of the parameters listed in Table 1 are needed to calibrate the model and to be set as reasonable values. The number of intervals $K$ was set to 9 , i.e., each interval lasted $10 \mathrm{~min}$, in order to reduce computational complexity. In reality, a minute-level comparison for departure time choice is not necessary, as users make their choices every 5 or 10 minutes. The two parameters of memory function $\left(y_{d-l}\right.$ and $L)$ were calibrated and explained in [29], while the parameters $\left(\alpha_{i}, \lambda_{i}\right.$, and $\left.\gamma\right)$ adopted in the prospect function were determined in [28]. The reference points $\left(t_{\mathrm{ea}}\right.$ and $\left.t_{\mathrm{la}}\right)$ were set according to the practical experience that users have little tolerance of arriving later and much more of being early.
The other parameters of the derived aggregate model (i.e., $\eta_{1}, \eta_{2}, \eta_{3}, \delta_{1}, \delta_{2}$, and $\delta_{3}$ ) have vital influence to the stable state and oscillation duration before equilibrium. Meanwhile, the value could be significantly different due to users' heterogeneity. In practical applications, these parameters needed to be calibrated using travel data from the specific region surveyed. In Table $1, \eta_{i}$ is the tolerance boundary of delay, set as a multiple of the current travel time. However, aiming to reveal the impact of $\eta_{i}$ and $\delta_{i}$, the results of different values are presented for a simple sensitivity analysis. The results are shown in Figures 5-8.

The above four figures show results for different parameter values. In order to simplify the discussion, we considered only one path and one departure slot for analysis. In Figures 5 and 6, we focus on parameter $\eta_{i}$ and set $\delta_{i}=0.5$, while in Figures 7 and $8, \eta_{i}=0.3$ and $\delta_{i}=0.2,0.4,0.6$.

It is obvious that a general rule cannot be readily extracted from the above figures. We can only conclude that larger tolerance and revision ratio corresponds to more consuming time and vigorous oscillation before reaching stability. This conclusion coincides with Corollary 2. However, if the two parameters $\eta_{i}, \delta_{i}$ are changed simultaneously, the impact of one will be offset by the other and this would invalidate the above law.

The results and analysis show that the equilibrium is not guaranteed in the proposed model for the expressions' discontinuity and undifferentiability, which is different from the fixed-point equilibrium or local stability for special parameters. The bounded rationality and experience reliance result in the solutions' multiplicity and final states' diversity.

\section{Conclusions}

In this study, we proposed a bounded-rational day-to-day dynamic learning and adjusting model that incorporates explicit expressions of individuals' spatial-temporary choice behaviours with navigation information. The establishment of the dynamical model started with individuals' information collection and self-learning along the time horizon, i.e., the travel time experienced and real-time navigation information collected on each discrete day. Then, a microscopic model was derived to determine the updating process of perceived travel time, the self-learning model, and the objective of maximizing utility in the system for each individual. Subsequently, the microscopic model was transformed to a macroscopic model to obtain a bounded-rational continuous day-to-day dynamical model by aggregating each individual's behaviour and calculating route choice probability. A numerical example indicates that the day-to-day model converges to a special stable state, but navigation reliance, risk preferences, the tolerance gap, and the initial state can all cause instabilities.

From the example results, it is obvious that navigation has a significant influence on traffic flow and the final equilibrium attained. Under this model, travellers showed more reliance on real-time information so that spatialtemporary flow manifested more drastic oscillations and a 
more concentrated departure time zone. The other most important elements were the tolerance gap $\left(\eta_{i}\right)$ and the adjustment probability $\left(\delta_{i}\right)$. These parameters will influence the model's oscillation, the oscillation's duration, and final convergence to stability. Furthermore, the numerical examples illustrated some important properties and indicated a compatible but different stable convergence from UE. The information collection pattern and users' past experience reliance will certainly affect day-to-day adjustment behaviour and changes in the final state. However, these differences are foreseeable, as the proposed dynamic model attached more importance to self-learning and reliance on experience, while UE pays more emphasis on the same utility on every option. The two models are suitable for various situations separately.

This study can be extended to deal with some unsolved issues. First, the dynamic model is based on the hypothesis that travellers can recollect their past travel time, the departure-time traffic state, and revise their day-to-day choices to a certain degree. When these assumptions change, for example, when users perceive only their own cost, navigation information is not precise enough or the revision rate varies among the users, and the model and result of the day-to-day stochastic fluctuations and final stability will change distinctly. Second, an initial normal distribution of flow is taken as first day choice data, which is appropriate for new road networks. However, if an unpredictable incident occurs on a network in use, the initial flow will be affected by various conditions and result in different final states. Third, the prospect computation algorithm in the proposed model emphasizes the choice result, where being late or early corresponds to disparate utility, but travel cost along the way is neglected. In fact, a large number of users will select their exact departure interval to avoid being too early or late, but for several participants, a too-early departure to enjoy a shorter travel time, less congestion, and lower fuel consumption could be a more appealing choice.

\section{Data Availability}

The data used to support the findings of this study are available from the corresponding author upon request.

\section{Conflicts of Interest}

The authors declare that they have no conflicts of interest.

\section{Acknowledgments}

This study was financially supported by the China Ministry of Education of Humanities and Social Science Project (19YJCZH007) and by the National Key Research and Development Program of China (2019YFB1600303).

\section{References}

[1] J. G. Wardrop, "Some theoretical aspects of road traffic research," Proceedings of the Institution of Civil Engineers, vol. 1, no. 3, pp. 325-362, 1952.
[2] C. F. Daganzo, "On the traffic assignment problem with flow dependent costs-II," Transportation Research, vol. 11, no. 6, pp. 439-441, 1977.

[3] Di. Xuan and H. X. Liu, "Boundedly rational user equilibria (BRUE): mathematical formulation and solution sets," Transportation Research Part B: Methodological, vol. 57, pp. 300-313, 2013.

[4] K. Han, W. Y. Szeto, and T. L. Friesz, "Formulation, existence, and computation of boundedly rational dynamic user equilibrium with fixed or endogenous user tolerance," Transportation Research Part B: Methodological, vol. 79, pp. 16-49, 2015.

[5] R. J. Smeed, M. Beckmann, C. B. Mcguire et al., "Studies in the economics of transportation," Economic Journal, vol. 26, no. 265 , pp. $820-821,1956$.

[6] J. L. Horowitz, "The stability of stochastic equilibrium in a two-link transportation network," Transportation Research Part B: Methodological, vol. 18, no. 1, pp. 13-28, 1984.

[7] M. J. Smith, "The stability of a dynamic model of traffic assignment-an application of a method of lyapunov," Transportation Science, vol. 18, no. 3, pp. 245-252, 1984.

[8] M. L. Hazelton, "Day-to-day variation in Markovian traffic assignment models," Transportation Research Part B: Methodological, vol. 36, no. 7, pp. 637-648, 2002.

[9] E. Cascetta, "A stochastic process approach to the analysis of temporal dynamics in transportation networks," Transportation Research Part B: Methodological, vol. 23, no. 1, pp. 1-17, 1989.

[10] D. P. Watling and G. E. Cantarella, "Modelling sources of variation in transportation systems: theoretical foundations of day-to-day dynamic models," Transportmetrica B: Transport Dynamics, vol. 1, no. 1, pp. 3-32, 2013.

[11] H.-J. Huang, T.-L. Liu, and H. Yang, "Modeling the evolutions of day-to-day route choice and year-to-year ATIS adoption with stochastic user equilibrium," Journal of Advanced Transportation, vol. 42, no. 2, pp. 111-127, 2008.

[12] I. Klein, N. Levy, and E. Ben-Elia, "An agent-based model of the emergence of cooperation and a fair and stable system optimum using atis on a simple road network," Transportation Research Part C: Emerging Technologies, vol. 86, pp. 183-201, 2018.

[13] F. Wei, N. Jia, and S. Ma, "Day-to-day traffic dynamics considering social interaction: from individual route choice behavior to a network flow model," Transportation Research Part B: Methodological, vol. 94, pp. 335-354, 2016.

[14] N. Gennaro, E. Giulio, and S. Fulvio, “Advanced traveller information systems under recurrent traffic conditions: network equilibrium and stability," Transportation Research Part B: Methodological, vol. 92, pp. 73-87, 2016.

[15] X. Li, W. Liu, and H. Yang, "Traffic dynamics in a bi-modal transportation network with information provision and adaptive transit serviceffic dynamics in a bi-modal transportation network with information provision and adaptive transit services," Transportation Research Part C: Emerging Technologies, vol. 91, pp. 77-98, 2018.

[16] W. Liu, X. Li, F. Zhang, and H. Yang, "Interactive travel choices and traffic forecast in a doubly dynamical system with user inertia and information provisionffic forecast in a doubly dynamical system with user inertia and information provision," Transportation Research Part C: Emerging Technologies, vol. 85, pp. 711-731, 2017.

[17] H. Ye, F. Xiao, and H. Yang, "Day-to-day dynamics with advanced traveller information," Transportation Research Part B: Methodological, vol. 44, p. 144, 2021. 
[18] D. B. Work, O. P. Tossavainen, S. Blandin et al., "An ensemble Kalman filtering approach to highway traffic estimation using GPS enabled mobile devices," 2008.

[19] S. Tao, V. Manolopoulos, S. Rodriguez, and A. Rusu, "Real-time urban traffic state estimation with A-GPS mobile phones as probes," Journal of Transportation Technologies, vol. 2, no. 1, pp. 22-31, 2012.

[20] J. C. Herrera, D. B. Work, R. Herring, X. Ban, Q. Jacobson, and A. M. Bayen, "Evaluation of traffic data obtained via GPS-enabled mobile phones: the Mobile Century field experiment," Transportation Research Part C: Emerging Technologies, vol. 18, no. 4, pp. 568-583, 2010.

[21] X. Guo and H. X. Liu, "Bounded rationality and irreversible network change," Transportation Research Part B: Methodological, vol. 45, no. 10, pp. 1606-1618, 2011.

[22] S. Chao, L. Menghui, C. Lin et al., "Boundedly rational user equilibrium with restricted unused routes," Discrete Dynamics in Nature and Society, vol. 34, pp. 1-11, 2016.

[23] M. B. Akiva, "Dynamic model of peak period congestion," Transportation Research Part B, vol. 18, no. 4, pp. 339-355, 1984.

[24] R.-Y. Guo, H. Yang, H.-J. Huang, and X. Li, "Day-to-day departure time choice under bounded rationality in the bottleneck model," Transportation Research Procedia, vol. 23, pp. 551-570, 2017.

[25] T. Iryo, "An analysis of instability in a departure time choice problem," Journal of Advanced Transportation, vol. 42, no. 3, pp. 333-358, 2008.

[26] R.-Y. Guo, H. Yang, and H.-J. Huang, "Are we really solving the dynamic traffic equilibrium problem with a departure time choice?" Transportation Science, vol. 52, no. 3, pp. 603-620, 2018.

[27] A. Tversky and D. Kahneman, "Advances in prospect theory: cumulative representation of uncertainty," Journal of Risk and Uncertainty, vol. 5, no. 4, pp. 297-323, 1992.

[28] D. Kahneman and A. Tversky, "Prospect theory: an analysis of decision under risk," Econometrica, vol. 47, no. 2, pp. 263-291, 1979.

[29] G. A. Miller, "The magical number seven, plus or minus two: some limits on our capacity for processing information," Psychological Review, vol. 63, no. 2, pp. 81-97, 1956.

[30] H. A. Simon, "How big is a chunk?: by combining data from several experiments, a basic human memory unit can be identified and measured," Science, vol. 183, no. 4124, pp. 482-488, 1974.

[31] M. Smith, M. L. Hazelton, H. K. Lo, G. E. Cantarella, and D. P. Watling, "The long term behaviour of day-to-day traffic assignment models," Transportmetrica A: Transport Science, vol. 10, no. 7, pp. 647-660, 2014.

[32] L. E. J. Brouwer, "Uber Abbildung von Mannigfaltigkeiten," Mathematische Annalen, vol. 71, no. 1, pp. 97-115, 1911. 